\title{
NÚMERO CROMOSÓMICO Y APAREAMIENTO MEIÓTICO EN TURBINICARPUS VALDEZIANUS (MÖLLER) GLASS \& FOSTER (CACTACEAE)
}

\author{
M. Humberto Reyes-Valdés \\ Martha Gómez-Martínez \\ Departamento de Fitomejoramiento \\ Universidad Autónoma Agraria Antonio Narro \\ 25315 Buenavista, Saltillo, Coahuila \\ Y \\ Hermila Trinidad García-Osuna
Estudiante de la Facultad de Ciencias Biológicas
Universidad Autónoma de Nuevo León
64000 San Nicolás de los Garza, Nuevo León

\begin{abstract}
RESUMEN
En este trabajo se estudiaron los cromosomas de Turbinicarpus valdezianus en diacinesis y metafase I, de plantas nativas de la región aledaña a Saltillo, Coahuila (México). Se encontró un número cromosómico $2 n=2 x=22$, lo cual es consistente con el número básico $x=11$ previamente conocido para otros miembros de la familia Cactaceae. El apareamiento normal bivalente observado en todas las células analizadas indica que la especie estudiada es diploide con una segregación cromosómica regular. El análisis de frecuencias de configuraciones meióticas apoya la hipótesis de que uno o más pares de cromosomas podrían ser no metacéntricos.
\end{abstract}

\section{ABSTRACT}

In this research the chromosomes of Turbinicarpus valdezianus were studied in diakinesis and metaphase I, from native plants located near Saltillo, Coahuila (Mexico). A chromosome number $2 n=2 x=22$ was found, which is consistent with the basic number $x=11$, previously reported for other members of the cactus family. The normal bivalent pairing observed in all the analyzed cells indicates that this is a diploid species with regular chromosome segregation. The analysis of meiotic configuration frequencies supports the hypothesis of one or more chromosome pairs being non-metacentric.

\section{INTRODUCCIÓN}

\section{Turbinicarpus valdezianus}

Turbinicarpus es un género de la familia de las cactáceas que incluye principalmente individuos globulares e inconspicuos. Sus frutos son bayas regularmente dehiscentes y, de 
acuerdo con Glass y Foster (1977), sus pequeñas espinas funcionan más bien como camuflaje que como estructuras repelentes a los depredadores. El taxon se encuentra en forma silvestre en México, distribuido a lo largo de la vertiente oeste de la Sierra Madre Oriental y sus planicies adyacentes en los estados de Coahuila, Nuevo León, Tamaulipas, San Luis Potosí, Zacatecas, Querétaro e Hidalgo (Hofer, 1995).

Las plantas de este género son muy apreciadas mundialmente como ornamentales y, a pesar de su lento crecimiento, son fáciles de cultivar (Cote, 1981). Todas las especies de Turbinicarpus están incluidas en el Apéndice I del CITES (Hunt, 1992). Además, las leyes mexicanas las protegen bajo la norma oficial NOM-059-ECOL-1994.

La reproducción en Turbinicarpus se lleva al cabo mediante semillas, cuya dispersión natural está determinada por hormigas, viento y corrientes de agua de lluvia. Ciertas especies, como T. valdezianus, T. pseudopectinatus y T. mandragora, exhiben un período de floración corto y bien definido. En la polinización intervienen insectos alados, aunque es posible observar hormigas en las flores (Hofer, 1995).

Turbinicarpus valdezianus comprende pequeñas plantas de 10 a $25 \mathrm{~mm}$ de diámetro (Fig. 1), aunque en invernadero pueden alcanzar mayores dimensiones. Sus tallos simples son deprimido-globosos a sub-cilíndricos, y producen una o más bellas flores de color blanco a violeta-rojizo. Su localidad tipo se encuentra en Coahuila, al noreste de la ciudad de Saltillo, donde fue originalmente descubierta y descrita por Möller en 1930 con el nombre de Pelecyphora valdeziana (Neutelings, 1982). Sin embargo, tal parece que la especie había sido brevemente descrita en 1892 en Europa por Schumann (Anton Hofer, comunicación personal). Después de ser ubicada en diferentes géneros es ahora reconocida como Turbinicarpus valdezianus (Möller) Glass \& Foster (Glass y Foster, 1977). En el área cercana a Saltillo las plantas presentan típicamente las flores de color violeta-rojizo. Fuera de esta localidad se registra principalmente la forma "albiflorus".

La especie en cuestión florece en los meses de enero y febrero, temporada del año en que la temperatura frecuentemente desciende a menos de $0^{\circ} \mathrm{C}$ en la región circundante a la ciudad de Saltillo. En esta área, el lugar de donde fue originalmente descrita la planta, ha sido parcialmente devastado por colectores, hasta que finalmente la población casi desapareció por la construcción de un aeropuerto. Adicionalmente, la expansión de caminos rurales, granjas avícolas y trituradoras de piedra han destruido una fracción considerable del resto de las poblaciones. Sin embargo, las plantas aún crecen en lomas poco accesibles para la actividad humana. De acuerdo con Anderson et al. (1994), la colecta representa el principal peligro para la supervivencia de la especie.

\section{Estudios cromosómicos y meióticos en cactáceas}

Los estudios cromosómicos ayudan a la clarificación de relaciones filogenéticas y taxonómicas. Con respecto a las cactáceas, Pinkava et al. (1992) afirman que la comparación del número y morfología cromosómica frecuentemente da lugar a identificación de linajes evolutivos previamente desconocidos. Además, cuando taxa de diferentes niveles de ploidía se hibridizan, pueden encontrarse ploidías intermedias en la progenie, por lo que los análisis cromosómicos son un valioso apoyo a las observaciones de fenotipos.

Muchas especies de cactáceas todavía no han sido analizadas citológicamente. Hasta el presente los resultados obtenidos coinciden con un número básico $x=11$; la única 
Reyes-Valdés et al.: Número Cromosómico y Apareamiento Meiótico en Turbinicarpus valdezianus

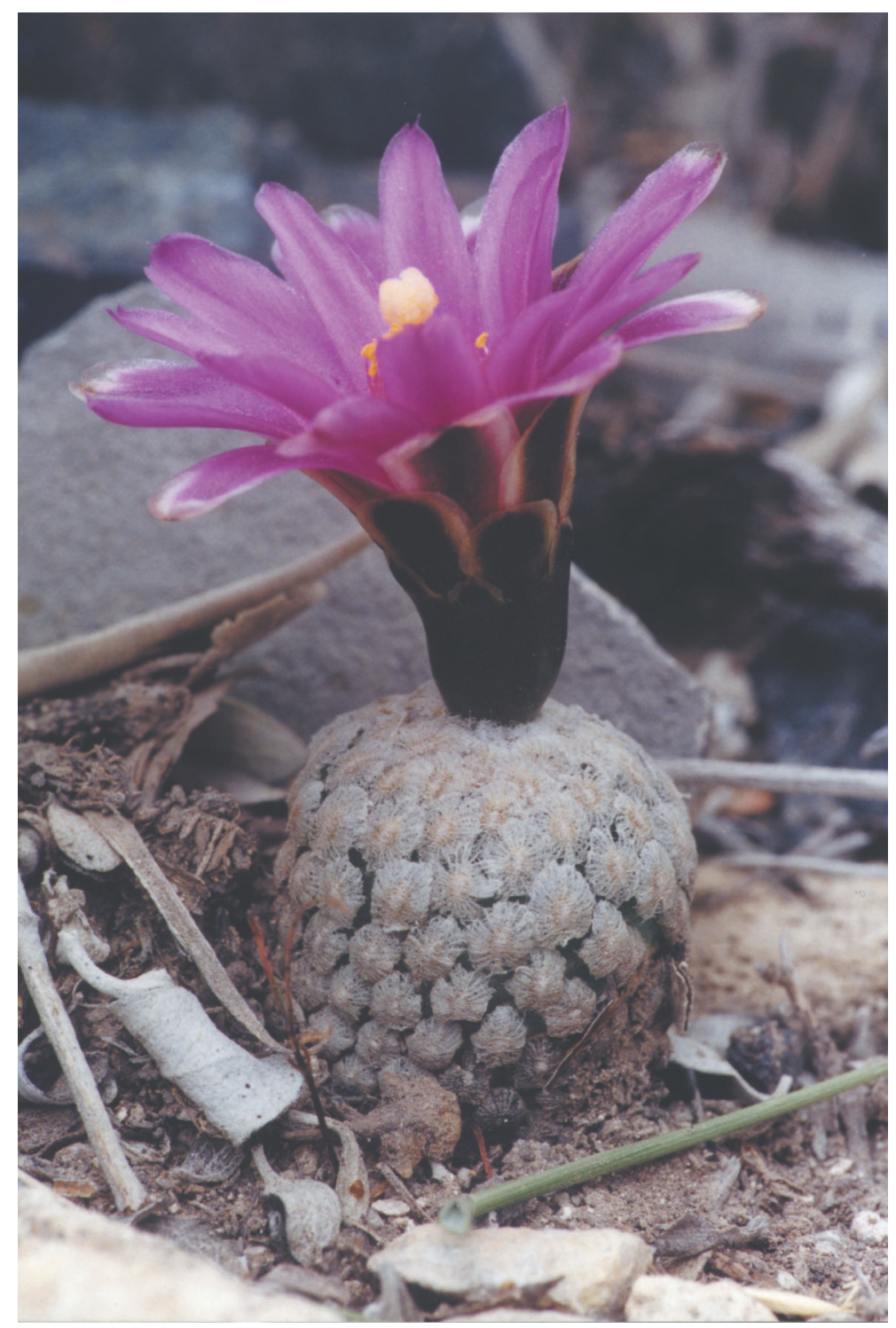

Fig. 1. Planta de $T$. valdezianus en plena floración en su hábitat natural. En este ejemplar el diámetro del tallo es de $14.3 \mathrm{~mm}$ y el de la flor de $19.4 \mathrm{~mm}$. 
excepción, que al parecer aún no ha sido confirmada, es el hallazgo de $x=12$ para Deamia testudo por Bhattacharyya (1970). Opuntia, el género más estudiado, forma una serie poliploide que incluye desde diploides $(2 n=2 x=22)$ hasta octaploides $(2 n=8 x=88)$. Tal parece que $O$. robusta tiene formas diploides y tetraploides, y sus números cromosómicos están relacionados con la dioecia (Granados y Castañeda, 1991).

En la subfamilia Cactoideae, la citología del género Mammillaria ha sido la más estudiada. El grupo presenta variación que va de los diploides con número cromosómico $2 n=2 x=22$, como M. polythele, hasta altos poliploides, como M. capensis con $24 x=264$ (Remski, 1954). Un caso interesante es el de $M$. prolifera, que tiene variedades con $2 n=$ $2 x=22,2 n=4 x=44$ y $2 n=6 x=66$ (Johnson, 1978).

El esfuerzo más importante para conocer los números cromosómicos de las cactáceas ha sido realizado por parte del equipo de Donald J. Pinkava, investigador de la Universidad Estatal de Arizona. A la fecha, se cuenta con una secuencia de siete publicaciones con estudios de representantes de esta familia en el oeste de Norte América (Pinkava y McLeod, 1971; Pinkava et al., 1973; Pinkava et al., 1977; Pinkava y Parfitt, 1982; Pinkava et al., 1985; Pinkava et al., 1992; Pinkava et al., 1998).

De acuerdo con Pinkava et al. (1998), se han contado los cromosomas de 650 taxa de cactáceas, comprendidos en 537 especies. $28.2 \%$ de los taxa presentan poliploidía, con el mayor porcentaje ubicado en Opuntiodeae. En esta subfamilia, de 199 taxa analizados $64.3 \%$ son poliploides. Para el caso de Cactoideae, con un total de 435 taxa analizados, solamente se ha registrado $12.9 \%$ de poliploidía, con la mayoría de los casos ubicados en Echinocereus y Mammillaria. De 56 géneros estudiados en esta subfamilia, 37 tienen sólo números diploides en sus especies. Por lo que respecta a la subfamilia Pereskioideae, los 14 taxa estudiados son todos diploides.

En el Royal Botanic Garden de Kew se está conformando una base de datos sobre valores $\mathrm{C}$ de $\mathrm{ADN}$ y números cromosómicos, que incluye información sobre varios taxa de cactáceas (Bennett et al., 1998); los géneros para los que se tienen incorporados números cromosómicos son: Aporocactus, Borzicactus, Cleistocactus, Escobaria, Mammillaria y Weberbaureocereus.

En cuanto a estudios de comportamiento meiótico en cactáceas, Johnson (1980) analizó algunas especies de Mammillaria. Encontró una meiosis regular en los taxa diploides, con 11 bivalentes en metafase I. Los tetraploides formaron 22 bivalentes en su mayoría, pero M. prolifera var. texana mostró algunos multivalentes. Por otro lado, Das et al. (1997) realizaron comparaciones de comportamiento meiótico entre especies de Mammillaria y encontraron una diversidad significativa en el número de quiasmas por célula, que varió de 19.42 en $M$. boolii a 28.80 en $M$. plumosa, ambas especies diploides $(2 n=2 x=22)$.

El objetivo del presente trabajo fue el conocer el número cromosómico de $T$. valdezianus con base en una población silvestre, así como analizar su forma de apareamiento meiótico. Se pretende así incrementar el conocimiento global de estos caracteres en cactáceas, al proveer datos del género Turbinicarpus, del cual no se encontró registro alguno en la literatura. Asimismo, se busca contribuir al estudio del comportamiento genético y reproductivo de $T$. valdezianus a través del análisis de su apareamiento cromosómico en la meiosis. 
Reyes-Valdés et al.: Número Cromosómico y Apareamiento Meiótico en Turbinicarpus valdezianus

\section{MATERIALES Y MÉTODOS}

Se obtuvieron botones florales de plantas nativas de una población silvestre de $T$. valdezianus con flores de color violeta-rojizo, localizada al noreste de Saltillo, Coah. El ejemplar de referencia que se utilizó para la identificación de las plantas (A. Flores $V$. 101) se encuentra en el herbario de la Universidad Autónoma Agraria Antonio Narro (ANSM). Los botones fueron fijados inmediatamente después de recolectados en una solución 3:1 (etanol: ácido acético glacial). Las anteras se extrajeron y maceraron en portaobjetos con colorante acetocarmín. Posteriormente, el material fue calentado por un minuto, aplastado bajo el cubreobjetos y observado con el objetivo 100x de un microscopio de luz. Los conteos cromosómicos se realizaron en células en diacinesis o metafase I de seis individuos de la población. Posteriormente, se seleccionaron por su calidad tres preparaciones, correspondientes a tres plantas distintas, para el estudio de configuraciones meióticas. De estas muestras se analizaron 22 células en diacinesis o metafase I en cuanto a configuraciones meióticas, mismas que se clasificaron en tres tipos: anillos, cadenas y pares univalentes, basándose en la presencia de quiasmas en ambos brazos, en un brazo o ausencia de quiasmas, respectivamente (Reyes-Valdés y Stelly, 1995; Reyes-Valdés et al., 1996). Las células en que no fue posible identificar claramente las configuraciones no se tomaron en cuenta para los registros de configuraciones, pero sí se usaron para corroborar el número cromosómico.

Con los datos de frecuencias de configuraciones meióticas, se sometió a prueba la hipótesis de que todos los pares homólogos tienen la misma probabilidad de formar cadenas, lo cual sería posible en el caso de que todos los cromosomas tuviesen aproximadamente el mismo tamaño y posición centromérica similar. Para ello se comparó la distribución de frecuencias del número de cadenas por célula, con una distribución de Poisson en la que el parámetro $\lambda$ fue igual al número promedio de cadenas por célula. Ya que esta distribución modela el caso en el que todos los pares homólogos tienen la misma probabilidad de formar cadenas, su similitud con las frecuencias observadas sería un indicio de homogeneidad en la morfología de los cromosomas dentro de cada una de las células.

La distribución observada de cadenas y la esperada de acuerdo con la distribución de Poisson fueron comparadas entre sí por medio del uso del índice varianza/media aplicado a los números observados de cadenas el cual, de acuerdo con Manly (1991), es un mejor instrumento estadístico que el uso de la prueba de bondad de ajuste por medio de $\chi^{2}$, especialmente para los casos en que existen clases con menos de cinco observaciones. La deficiencia del índice varianza/media es la ausencia de fórmulas para calcular su intervalo de confianza; sin embargo, es posible usar el método de remuestreo denominado "bootstrap" para determinar dicho espacio (Press et al., 1992). En este trabajo se realizaron 10000 remuestreos para obtener el intervalo de confianza de $95 \%$ del índice varianza/media.

\section{RESULTADOS Y DISCUSIÓN}

En todas las células analizadas de las seis plantas se observaron 11 bivalentes, como lo muestran las Figuras $2 \mathrm{~A}$ y $2 \mathrm{~B}$, donde se presentan microfotografías de células en diacinesis. Lo anterior indica que $T$. valdezianus es diploide, con un número cromosómico 
$2 n=2 x=22$. Aunque no se descarta la posibilidad de encontrar un nivel de ploidía diferente en otra población, o en la forma "albiflorus", esto parece muy poco probable, porque en la subfamilia Cactoideae la presencia de números diferentes de cromosomas dentro de la misma especie es algo excepcional. Por otro lado, se sabe que las desigualdades en pigmentación en las plantas se deben por lo general a diferencias alélicas y no de número cromosómico.

El patrón de apareamiento meiótico bivalente observado permite una segregación cromosómica regular y en consecuencia un comportamiento reproductivo sexual. De acuerdo con nuestras observaciones, la reproducción sexual parece ser el patrón normal en esta especie, porque su propagación se lleva al cabo a través de semillas. Por otro lado, ya que la apomixis se vincula usualmente con plantas poliploides, e incluso pudiera estar correlacionada con la tetrasomía (Grimanelli et al., 1998), es muy poco probable un origen apomíctico de las semillas de $T$. valdezianus.

En la Figura 2B se muestra una de las células que se usaron en el análisis de configuraciones meióticas, donde se señala una cadena bivalente por medio de una flecha, mientras que los diez bivalentes restantes corresponden a anillos. En las 22 células analizadas se registró $80.2 \%$ de anillos, $19.8 \%$ de cadenas y no se observaron pares univalentes. El promedio de cadenas por célula fue de 2.18. La ausencia de univalentes es un buen indicador de que la segregación cromosómica es balanceada.
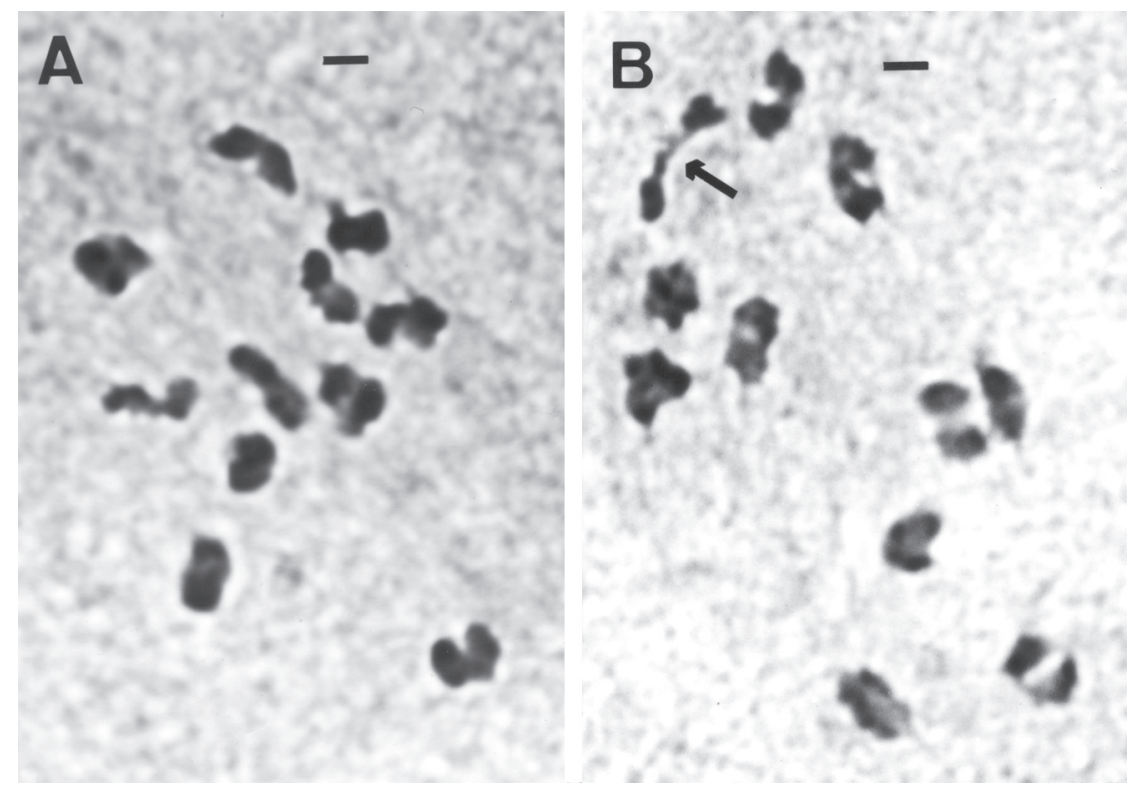

Fig. 2. Diacinesis en T. valdezianus con un total de 11 bivalentes. A. Conjunto de bivalentes muy condensados, en el cual es fácil detectar el número cromosómico, pero no se pueden identificar las configuraciones con seguridad. B. Conjunto de bivalentes cuyo tipo de configuración es identificable y corresponde al tipo de anillos, con excepción de la cadena señalada por una flecha. La longitud de la escala en la parte superior de cada fotografía es de $2 \mathrm{~m}$. 
En la Figura $3 \mathrm{~A}$ se presenta el histograma de frecuencias absolutas observadas de células para cada número de cadenas y en la Figura $3 B$ se presenta el histograma correspondiente a las frecuencias esperadas de acuerdo con una distribución de Poisson con parámetro $\lambda=2$.18. Puede notarse la ausencia de células con cero cadenas y el exceso de las que contienen una cadena en los datos experimentales, al ser comparados con lo esperado en función de la hipótesis de homogeneidad de probabilidades de formación de cadenas en los diferentes pares homólogos.

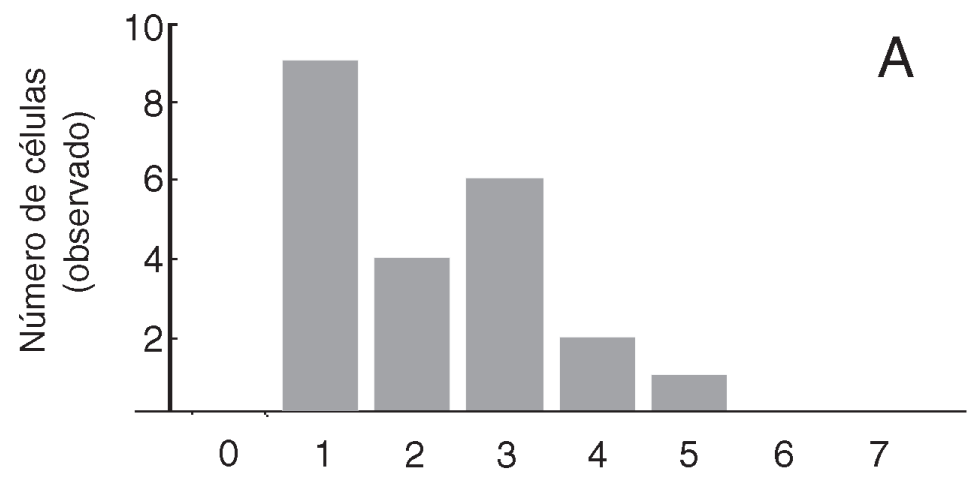

Número de cadenas

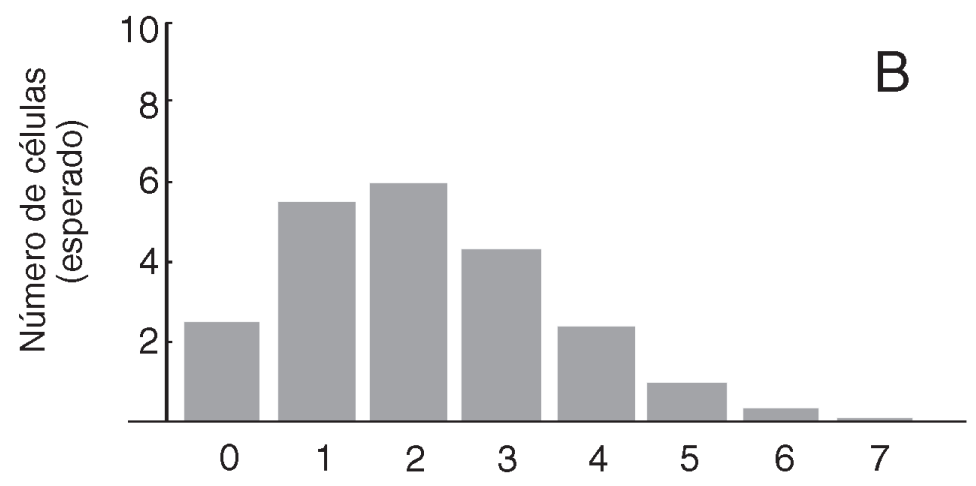

Número de cadenas

Fig. 3. A. Histograma de frecuencias absolutas observadas del número de cadenas bivalentes por célula. B. Histograma de frecuencias absolutas esperadas bajo la suposición de que todos los cromosomas tienen la misma probabilidad de formar cadenas. 
El intervalo de confianza de 95\% generado por medio de remuestreo para el índice varianza/media, aplicado al número de cadenas por célula, resultó ser de $(0.39,0.95)$. El hecho de que todo el intervalo se encuentra dentro de (varianza/media) $<1$, es indicativo de una distribución experimental que no se ajusta a la de Poisson, porque el número de cadenas por célula es más uniforme de lo esperado. Estos resultados parecen sugerir que existe uno o más pares de cromosomas no metacéntricos, que regularmente forman cadenas en el apareamiento homólogo. Como se sabe, la morfología cromosómica es un parámetro que puede ser utilizado en la comparación entre taxa, sin embargo para tales propósitos el examen de cromosomas en metafase mitótica es más adecuado que los estudios efectuados al nivel de la meiosis. Por ejemplo, en el análisis de mitosis de Mammillaria prolifera se ha podido observar que la mayoría de los cromosomas son metacéntricos con aproximadamente la misma longitud, sin embargo, existe alguna variación en la posición del centrómero, especialmente en la forma tetraploide, el cual posee cinco pares acrocéntricos (Johnson, 1980).

\section{CONCLUSIONES}

Los resultados indican que Turbinicarpus valdezianus es diploide, con número cromosómico $2 n=2 x=22$, el cual es consistente con el número básico $x=11$, definido para las cactáceas estudiadas hasta el presente. Por otro lado, el carácter $2 x=22$ coincide con la mayoría de los componentes estudiados de la subfamilia Cactoideae. La naturaleza diploide de las plantas analizadas y su apareamiento cromosómico bivalente son buenos indicadores de que la formación de su semilla tiene origen sexual. El estudio de configuraciones meióticas indica que, al menos para la población estudiada, el cariotipo podría incluir uno o más pares de cromosomas no metacéntricos.

\section{AGRADECIMIENTOS}

Agradecemos los valiosos comentarios del Sr. Anton Hofer, así como las facilidades que nos otorgó para hacer uso de su acervo bibliográfico. Este trabajo se llevó al cabo en la Universidad Autónoma Agraria Antonio Narro, con fondos proveídos por la Comisión Nacional para el Conocimiento y Uso de la Biodiversidad (CONABIO), a través del proyecto R007.

\section{LITERATURA CITADA}

Anderson, E. F., S. Arias y N. P. Taylor. 1994. Threatened cacti of Mexico. Succ. Plant Res. 2: 94115.

Bennett, M. D., A. V. Cox y I. J. Leitch. 1998. Angiosperm DNA C-values database. http:// www.rbgkew.org.uk/cval/database.html.

Bhattacharyya, P. K. 1970. Cytological study of Deamia testudo (Karw). Britt. \& Rose: A cactus growing wild in West Bengal. Sci. \& Culture 36:108.

Cote, D. 1981. Turbinicarpus: growing tiny plants for a large reward. Cact. Succ. J. (USA) 53: 244245. 
Reyes-Valdés et al.: Número Cromosómico y Apareamiento Meiótico en Turbinicarpus valdezianus

Das, A. B., S. Mohanty y P. Das 1997. Meiotic behavior and nuclear DNA variation in some species of Mammillaria (Cactaceae). Cytologia 62: 253-257.

Glass, C. y R. Foster. 1977. A revision of the genus Turbinicarpus (Backbg.) Buxb. \& Backbg. Cact. Succ. J. (USA) 49: 161-176.

Granados, D. y A. D. Castañeda. 1991. El nopal. Editorial Trillas. México, D.F. 227 pp.

Grimanelli, D., O. Leblanc, E. Espinosa, E. Perotti, D. González de León y Y. Savidán. 1998. Mapping diplosporous apomixis in tetraploid Tripsacum: one gene or several genes? Heredity 80: 3339.

Hofer, A. 1995. Quelques considérations sur le genre Turbinicarpus (Backeberg) Buxbaum et Backeberg. Succulentes (Francia) 18: 22-29.

Hunt, D. 1992. CITES Cactaceae checklist. Royal Botanic Gardens. Kew, Surrey. 190 pp.

Johnson, M. A. T. 1978. Diploid cytotypes in Mammillaria prolifera and three other Mammillaria species. Cact. Succ. J. Gr. Brit. 40: 9-12.

Johnson, M. A. T. 1980. Further cytological investigations in Mammillaria prolifera and other Mammillaria species. Cact. Succ. J. Gr. Brit. 42: 43-47.

Manly, B. F. J. 1991. Randomization and Monte Carlo methods in biology. Chapman and Hall. Nueva York. $281 \mathrm{pp}$.

Neutelings, T. M. W. 1982. Turbinicarpus valdezianus (Möller) Gl. \& F. Succulenta (Amsterdam) 61: 188-190.

Pinkava, D. J., M. A. Baker, B. D. Parfitt, M. W. Mohlenbrock y R. D. Worthington. 1985. Chromosome numbers in some cacti of western North America-V. Syst. Bot. 10: 471-483.

Pinkava, D. J., L. A. Mc Gill, T. Reeves y M.G. McLeod. 1977. Chromosome numbers in some cacti of western North America-III. Bull. Torrey Bot. Club 104: 105-110.

Pinkava, D. J. y M G. McLeod. 1971. Chromosome numbers in some cacti of western North America. Brittonia 23: 171-176.

Pinkava, D. J., M. G. McLeod, L. A. McGill y R. C. Brown. 1973. Chromosome numbers in some cacti of western North America-II. Brittonia 25: 2-9.

Pinkava, D. J. y B. D. Parfitt. 1982. Chromosome numbers in some cacti of western North AmericaIV. Bull. Torrey Bot. Club 109: 121-128.

Pinkava, D. J., B. D. Parfitt, M. A. Baker y R. D. Worthington. 1992. Chromosome numbers in some cacti of western North America-VI. Madroño 39: 98-113.

Pinkava, D. J., J. P. Rebman y M. A. Baker. 1998. Chromosome numbers in some cacti of western North America-VII. Haseltonia 6: 32-41.

Press, W. H., S. A. Teukolsky, W. T. Vetterling y B. P. Flannery. 1992. Numerical recipes in fortran. 2a. ed. Cambridge University Press. Cambridge. 963 pp.

Remski, M. F. 1954. Cytological investigations in Mammillaria and some associated genera. Bot. Gaz. 116: $163-171$.

Reyes-Valdés, M. H. y D. M. Stelly. 1995. A maximum likelihood algorithm for genome mapping of cytogenetic loci from meiotic configuration data. Proc. Natl. Acad. Sci. USA 92: 9824-9828.

Reyes-Valdés, M. H., Y. Ji, C. F. Crane, J. F. Taylor, M. N. Islam-Faridi, H. J. Price y D. M. Stelly. 1996. ISH-facilitated analysis of meiotic bivalent pairing. Genome 39: 784-792.

Schumann, K. 1892. Eine dritte Spezies von Pelecyphora. Monatsschrift für Kakteenkunde (Berlin) 2: 44. 